\title{
Couches minces fabriquées par sol-gel pour des applications en optique non linéaire
}

\author{
J. Fick, A. Martucci* et M. Guglielmi* \\ Laboratoire d'Électromagnétisme Micro-ondes et Optoélectronique, UMR 5530, \\ INPG-UJF-CNRS, 23 rue des Martyrs, BP. 257, 38016 Grenoble, France \\ * Dipartimento di Ingegneria Meccanica, Settore Materiali, Universita di Padova, \\ via Marzolo 9, 35131 Padova, Italy
}

\begin{abstract}
Résumé : Des couches minces de $\mathrm{SiO}_{2}-\mathrm{TiO}_{2}$ dopées avec des nanocristallites de $\mathrm{PbS}$ ont été fabriquées par sol-gel. La présence des nanocristallites a été mise en évidence par des spectres d'absorption et des clichés de MEB. L'indice de réfraction non linéaire a été mesuré par la technique des lignes noires non linéaires et le mélange à quatre ondes. Des valeurs de $n_{2}$ entre $-10^{-7}$ et $-10^{-10}$ $\mathrm{cm}^{2} / \mathrm{kW}$ ont été obtenues en fonction du taux de dopage et de la durée des impulsions laser utilisées. Des effets de saturation de la non-linéarité ont été observés. Le temps de réponse de la non-linéarité mesuré est inférieur à 30 ps.
\end{abstract}

\section{INTRODUCTION}

Un grand effort de recherche est actuellement fait sur les nanostructures de semi-conducteur ou de métal. Elles présentent un très grand intérêt pour les applications en optique intégrée, par exemple pour la commutation tout optique [1] ou la propagation des solitons optiques [2]. Beaucoup de travaux ont été faits sur des verres dopés avec des nanocristallites de CdS et $\mathrm{CdSeS}$ [3], principalement à cause de leur disponibilité comme filtres optiques commerciaux. Une des limitations principales de ces matériaux est le photonoircissement, c'est-à-dire le changement permanent des propriétés optiques sous irradiation laser. Il a été démontré que ce problème peut être résolu en utilisant la technique sol-gel pour la fabrication des verres [4].

Un autre dopant très prometteur est le $\mathrm{PbS}$. Sa bande d'absorption sous forme massive est située aux alentours de $3 \mu \mathrm{m}$. Elle peut être décalée vers le visible par confinement quantique. Dans le cas d'un faible élargissement inhomogène de la transition excitonique, de fortes nonlinéarités sont prévues [5]. Des nanocristallites de PbS ont été fabriqués dans les verres [6], les polymères [7], en solution [8] ou par sol-gel [9]. La plupart des auteurs ont observé le décalage vers le bleu de la bande d'absorption, mais, à notre connaissance, il n'y a pas de valeurs publiées de l'indice de réfraction non linéaire.

\section{FABRICATION DES ÉCHANTILLONS}

Des couches minces de $\mathrm{SiO}_{2}-\mathrm{TiO}_{2}$ contenant des nanocristallites de $\mathrm{PbS}$ sont fabriquées en utilisant la technique de sol-gel. Le dopage est réalisé par la préparation séparée du sol de la matrice et du sol du dopant [10]. Cette méthode permet d'obtenir une très faible dispersion de 
taille des cristallites. Les nanocristallites sont obtenus par la réaction de l'acétate de plomb avec du thioacétamide dans une solution de méthanol qui contient un agent de passivation de surface. La taille des cristallites est contrôlée par la concentration de cet agent. Le sol de la silice est obtenu par mélange de tétraéthoxysylane (TEOS) et de méthyltriéthoxysilane (MTES) dans de l'éthanol avec de l'eau et du HCl. Les deux solutions sont mélangées et le sol du $\mathrm{TiO}_{2}$ est ensuite ajouté. Des couches minces sont obtenues par "dip coating" sur un substrat en silice fondue. Un traitement thermique à $300^{\circ} \mathrm{C}$ pendant une heure sous atmosphère d'azote assure l'évacuation des solvants et la densification de la couche. Pour obtenir des couches minces d'une épaisseur d'environ $1 \mu \mathrm{m}$, le dépôt successif de 4 à 6 couches est nécessaire. Des couches minces de $70 \mathrm{~mol}^{\circ} \mathrm{SiO}_{2}$ et $30 \mathrm{~mol} \% \mathrm{TiO}_{2}$ dopées avec 5 à $25 \mathrm{~mol} \%$ $\mathrm{PbS}$ ont été fabriquées pour la caractérisation linéaire et non linéaire. Toutes les couches supportent au moins un mode guidé à la longueur d'onde de mesure $(1.064 \mu \mathrm{m})$.

Les spectres d'absorption linéaire des couches minces dopées avec du $\mathrm{PbS}$ montrent une bande d'absorption aux alentours de $1.4 \mu \mathrm{m}$ (figure 1). La raie d'absorption de la première transition excitonique est visible à $1.2 \mu \mathrm{m}$. Cette raie peut être modélisée par un calcul qui prend en compte l'élargissement inhomogène de la raie dû à la distribution de taille des cristallites. Pour calculer la bande d'absorption en fonction de la taille de cristallites, nous utilisons le modèle des bandes hyperboliques [7]. L'accord entre les courbes expérimentale et théorique est bon (figure 1). Pour l'échantillon dopé avec $10 \%$ de $\mathrm{PbS}$, nous obtenons une taille moyenne de $5.3 \mathrm{~nm}$ avec une dispersion de taille de $13 \%$. Ces valeurs sont en bon accord avec les observations faites au microscope électronique à balayage (MEB). Cette faible distribution de taille est obtenue grâce à la préparation séparée des cristallites et de la matrice.

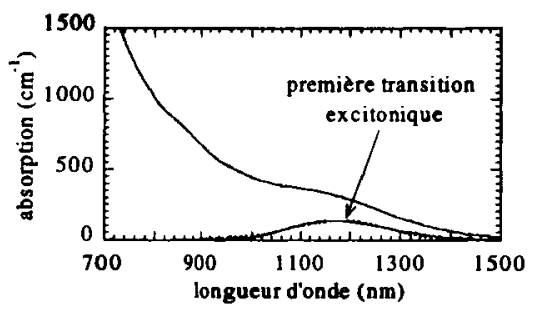

Figure 1 : Spectre d'absorption optique d'un échantillon avec 10\% de PbS. Les deux courbes de la transition excitonique correspondent a la mesure et au calcul thérique respectivement.

\section{CARACTÉRISATION NON LINEAIRE}

Les propriétés non linéaires ont été mesurées par deux méthodes différentes qui sont bien adaptées aux mesures de couches minces : les lignes noires non linéaires (LN) et le mélange à quatre ondes (DFWM). Les deux techniques permettent de mesurer la valeur absolue de l'indice de réfraction non linéaire $\mathrm{n}_{2}$. Le signe de $\mathrm{n}_{2}$ peut être déterminé par les lignes noires et le temps de réponse par le DFWM. Nous utilisons différents lasers Nd:YAG à $1.064 \mathrm{~mm}$ en régime nanoseconde (LN: $10 \mathrm{~ns} / 10 \mathrm{~Hz}$ ) et picoseconde mono-coup (LN: $60 \mathrm{ps} / 10 \mathrm{~Hz}$, DFWM: $35 \mathrm{ps} / 5 \mathrm{~Hz})$.

Pour les mesures de lignes noires non linéaires, un prisme à haut indice (SF58) est utilisé pour coupler le faisceau laser dans les couches minces. Dans l'expérience, l'intensité réfléchie est enregistrée en fonction de l'angle d'incidence [11]. Pour les angles de résonance, une partie de l'énergie incidente est couplée dans le guide d'onde et nous observons une ligne noire en 
réflexion. Les lignes noires enregistrées sur un échantillon contenant $20 \%$ de $\mathrm{PbS}$ sont représentées sur la figure $2 \mathrm{a}$. Les deux courbes sont enregistrées respectivement à basse et à haute intensité du faisceau incident. Le décalage vers les plus petits angles de la courbe enregistrée à haute puissance par rapport à celle enregistrée à basse puissance indique un effet non linéaire négatif. L'utilisation d'un faisceau de largeur limitée mène à une distribution inhomogène de l'intensité guidée et par conséquence à des conditions de couplages inhomogènes, ce qui explique la diminution du contraste observée sur la figure 2a. À partir d'un modèle théorique du coupleur distribué [12], nous déduisons l'indice de réfraction non linéaire de ces mesures. La réversibilité de l'effet non linéaire est vérifiée en enregistrant une deuxième courbe à basse intensité, après la mesure à haute intensité. Pour les échantillons fabriqués par sol-gel, nous n'avons pas observé d'effets irréversibles. Les effets du photonoircissement dans un verre dopé classique (Schott OG 530) ont été, néanmoins bien visibles.

Tableau 1: Résumé des résultats non linéaires en $\left[\mathrm{cm}^{2} / \mathrm{kW}\right]$.

\begin{tabular}{lcccccc}
\hline \multicolumn{2}{l}{ Taux de dopage } & $5 \%$ & $10 \%$ & $15 \%$ & $20 \%$ & $25 \%$ \\
\hline lignes noires & $10 \mathrm{~ns}$ & $-210^{-8}$ & $-310^{-8}$ & $-110^{-7}$ & $-810^{-8}$ & $-110^{-7}$ \\
& $60 \mathrm{ps}$ & - & $-110^{-10}$ & $-210^{-10}$ & $-410^{-10}$ & $-210^{-9}$ \\
DFWM & $35 \mathrm{ps}$ & - & - & - & $810^{-10}$ & $110^{-9}$ \\
\hline
\end{tabular}

Les résultats des mesures de $\mathrm{n}_{2}$ sont résumés dans le tableau 1. En régime nanoseconde nous avons mesuré de fortes non-linéarités négatives de $10^{-8}$ à $10^{-7} \mathrm{~cm}^{2} / \mathrm{kW}$. Ces valeurs sont du même ordre de grandeur que pour d'autres nanocristallites de semi-conducteurs [13,14]. En régime picoseconde, les valeurs sont typiquement 100 fois plus petites. Ceci peut être expliqué par la saturation de la non-linéarité. La non-linéarité dans des nanocristallites peut en effet être décrite par la saturation de l'absorption d'un système à deux niveaux. Ce modèle prévoit la saturation de la non-linéarité pour de très fortes intensités. Or l'intensité crête de l'impulsion laser en régime nanoseconde est typiquement $10-40 \mathrm{MW} / \mathrm{cm}^{2}$, soit cent fois plus petite qu'en régime picoseconde $\left(1000-4000 \mathrm{MW} / \mathrm{cm}^{2}\right)$. En régime nanoseconde, nous avons observé une non-linéarité non saturée. Mais en régime picoseconde les effets de saturation sont bien visibles.

Dans le cas du mélange à quatre ondes, deux faisceaux de pompe interfèrent sur l'échantillon et créent un réseau de diffraction par l'intermédiaire de l'effet non linéaire [11]. L'efficacité de diffraction de ce réseau est mesurée par un faisceau sonde. L'indice de réfraction non linéaire peut ensuite être calculé par le modèle du réseau mince. Le signal n'a été assez fort pour déterminer $n_{2}$ que pour les deux échantillons les plus fortement dopés (tab. 1). Les valeurs sont en bon accord avec les résultats obtenus par les lignes noires. Sur la figure $2 b$ nous montrons l'intensité diffractée de la sonde en fonction de son retard par rapport aux faisceaux de pompe pour un échantillon dopé avec $20 \%$ de $\mathrm{PbS}$. La forme symétrique du pic indique un temps de réponse de la non-linéarité plus court que la durée de l'impulsion laser (30 ps). Comme dans ce cas d'excitation résonante l'effet non linéaire est lié à des transitions réelles, nous en déduisons que le temps de recombinaison des porteurs libres est inférieur à 30 ps. Dans les semi-conducteurs massifs, ce temps est typiquement de l'ordre de la dizaine de nanosecondes. La recombinaison est donc beaucoup plus rapide dans les nanocristallites. Ce fait a été déjà observé par d'autres équipes dans les verres dopés de CdSSe [15]. L'influence du photonoircissement [16] et de l'effet Auger [17] a été démontrée dans ces matériaux. 

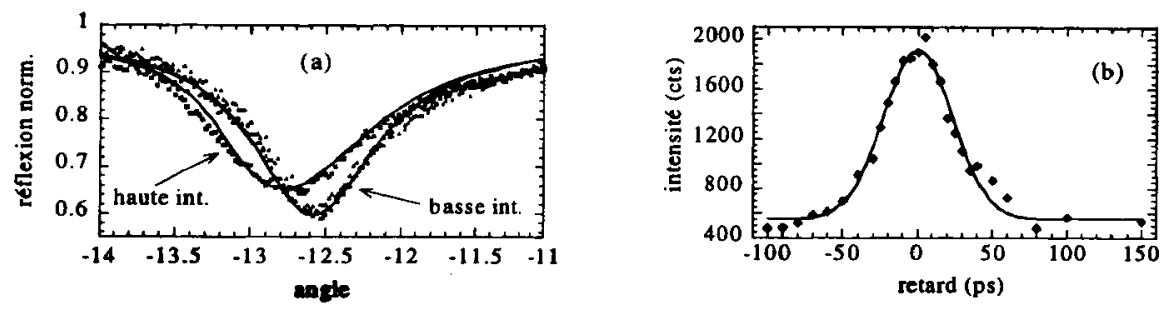

Figure 2 : Résultats non linéaires. (a) Lignes noires d'un échantillon contenant $15 \%$ de PbS, enregistrées à basse et haute intensité. Les points correspondent aux mesures et les traits continus au calcul théorique. (b) Intensité diffractée dans l'expérience du mélange à quatre ondes pour un échantilion avec $20 \%$ de PbS.

\section{CONCLUSION}

La technique de sol-gel permet la fabrication de couches minces vitreuses et dopées avec des nanocristallites de semi-conducteur. Le dopage avec $\mathrm{PbS}$ engendre des effets non linéaires très intéressants avec un grand potentiel pour des études futures.

\section{Références}

[1] Ironside C. N., Contemp. Phys. 34 (1993) 1-18.

[2] F. Kajzar et R. Reinisch, Beam Shaping and Control with Nonlinear Optics (Plenum Press, New York, 1998) p. 183-290.

[3] par ex. Banfi G. P., Degiorgio V. et Tan H. M., J. Opt. Soc. Am. B 12 (1995) 621-628.

[4] Kang K., Kepner A. D., Hu Y.Z., Koch S. W., Peyghambarian N., Li C.-Y., Takada T., Kao Y. et Mackenzie J. D., Appl. Phys. Lett. 64 (1994) 1487-1489.

[5] Banyai L., Hu Y. Z., Lindberg M. et Koch S. W., Phys. Rev. B 38 (1988) 8142-8153.

[6] Borelli N. F. et Smith D. W., J. Non-Cryst. Solids 180 (1994) 25-31.

[7] Wang Y., Suna A., Mahler W. et Kasowski R., J. Chem. Phys. 87 (1987) 7315-7322.

[8] Nenadocic N. T., Comor M. I., Vasic V. et Macic O. I., J. Phys. Chem. 94 (1990) 63906396.

[9] Nogami M., Nagasaka K. et Kotani K., J. Non-Cryst. Solids 126 (1990) 87-92.

[10] Guglielmi M., Martucci A., Menegazzo E., Righini G. C., Pelli S., Fick J. et Vitrant G., J. Sol Gel Science and Techn. 8 (1997) 1017-1021.

[11] Fick J., Schell J., Lévy R., Martucci A. et Guglielmi M., Pure Appl. Opt. 6 (1997) 527535.

[12] Vitrant G., Reinisch R., Kajzar F. Opt. Eng. 34 (1995) 3418-3427.

[13] Fick J., Vitrant G., Martucci A., Guglielmi M., Pelli S. et Righini G. C , Nonlin. Opt. 12 (1995) 203-209.

[14] Yumoto J., Fukushima S. et Kubodera K., Opt. Lett. 12 (1987) 832-833.

[15] par ex. Cotter D. Ironside C. N., Ainslie B. J. et Girdlestone H. P., Opt. Lett. 14 (1989) p. 317-319.

[16] Roussignol P., Ricard D., Lukasik J. et Flytzanis C. J. Opt. Soc. Am B 4 (1987) 5-13.

[17] Ghanassi M., Schanne-Klein M. C., Hache F., Ekimov A. I., Ricard D., Flytzanis C., Appl. Phys. Lett. 62 (1993) 78-80. 